\title{
Advanced Elbow Joint Tuberculosis with Cervicothoracic Junction Extramedullary Tuberculoma in a School Boy: Unusual Association
}

Sir,

Tuberculosis constitutes a global health concern, however, with increase in cases of HIV cases; there is also likely concurrent rise in incidence of cases suffering with tuberculosis. However, tuberculosis is endemic in developing country. The lung is the primary site of tuberculosis and skeletal system involvement is observed in approximately $1 \%-3 \%$ of the patients. ${ }^{[1-3]}$ Vertebral involvement is considered as the most common site of skeletal system involvement. Skeletal extraspinal tuberculosis involvement is usually noted only in hip, knee, foot, and shoulder joints. ${ }^{[4-7]}$ Tuberculosis affecting the joints of upper limb is rare and elbow joint tuberculosis is uncommon but reported. ${ }^{[8-11]}$ Tuberculous arthropathy is a type of musculoskeletal manifestation of tuberculosis and a common cause of infectious arthritis in developing countries and usually represents reactivation of primary pulmonary or lymph node tuberculosis. Tubercular arthropathy can occur at any age; however, no sex predilection is observed. The primary causative organism is Mycobacterium tuberculosis; however, atypical mycobacteria can affect in immunocompromised patients. Usually, M. tuberculosis remains dormant until recrudescence occurs. ${ }^{[12]}$ Tuberculosis involvement of elbow joint is extremely uncommon and about thirty cases are reported in the literature. Charcot arthropathy may rarely be sequelae of tuberculous arthropathy, characterized by dislocations of joint, pathological joint fractures, causing profound debilitating deformities. It results due to progressive destruction of bone and joint soft tissues, preferably affecting large weight bearing joints, and in the advanced stage may cause significant disruption of the bony architecture. Author reports an interesting case of advanced elbow joint tuberculosis occurring in a 16-year boy, who also had intradural-extramedullary mass of tuberculosis origin at cervicothoracic junction.

A 16-year-old boy presented with a complaint of insidious onset weakness and wasting of hand muscle for 6 months, along with difficulty in walking. He was diagnosed as a case of open pulmonary tuberculosis 8 months back, kept on regular antitubercular medication comprising rifampicin, isoniazid mycobutol, and pyrazinamide for initial 2 months, and later on, he was kept on only two drugs therapy. Despite continuation of antituberculous medication, he noticed progressive swelling and deformity evaluated by orthopedician and diagnosed as advanced cases of peripheral osteoarticular tuberculosis of left elbow joint with ankylosis. Local examination of elbow joint revealed swelling and loss of elbow contour and fixed contracture with completely immobile elbow joint. Neurological examination revealed gross wasting of thenar and hypothenar muscle with weakness of intrinsic hand muscles and lower limb power was $4 / 5$ with bilateral brisk knee and ankle jerks with bilateral ill-sustained patellar and ankle clonus and bilateral plantar extensor response. Sensory evaluation revealed bilateral grade sensory loss below D6 dermatome, asymmetrically distributed.

X-ray elbow joint revealed destruction of olecranon process of ulna with destruction of condyles with gross Charcot's arthropathy [Figure 1]. Magnetic resonance imaging (MRI) dorsal spine revealed that the presence of intradural-extramedullary mass comprised with multiple conglomerate ring lesions at C7-D1 level situated on left lateral aspect, measuring about $10 \mathrm{~mm} \times 14 \mathrm{~mm}$ in dimension, causing displacement and compression of spinal cord [Figures 2 and 3], showing hyperintense signal on T2-weighted image and iso on T1 image and with homogeneous contrast enhancement with associated meningeal enhancement and the presence of incidental hemangioma of fourth dorsal vertebra.

He underwent C7-D1 laminoplasty with excision of intradural mass, the lesion was grayish with ill-defined plane of cleavage and gross total excision was carried out. The histopathological examination was compatible with caseating tuberculous lesion. He was also continued on the second-line antituberculous medication in view of multidrug resistance tuberculosis and presently awaiting arthrodesis of elbow joint.

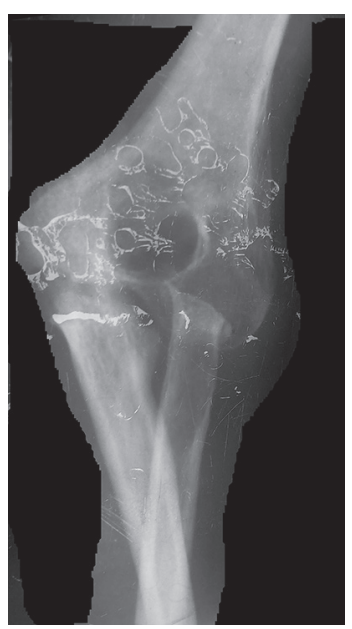

Figure 1: X-ray anteroposterior view of elbow joint showing complete destruction of elbow joint 


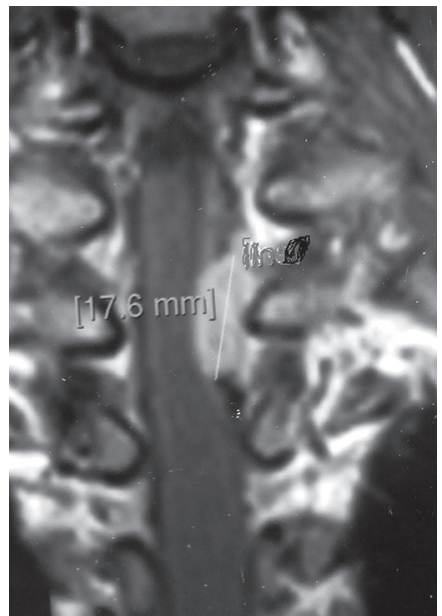

Figure 2: Magnetic resonance imaging cervicodorsal spine, gadoliniumenhanced study, sagittal section image showing tuberculoma at C7-D1 cervicothoracic spine junction showing conglomerate ring enhancement

Tuberculosis can be classified as either pulmonary or extrapulmonary, while the bone and joint tuberculosis may account for approximately $35 \%$ of extrapulmonary tuberculosis. ${ }^{[1]}$ Osteoarticular tuberculosis includes spinal tuberculosis $(50 \%)$ and remainder affects bone bearing joint, i.e., hip and knee, and another $15 \%$ being polyarticular but nonweight bearing joint affection of elbow is very infrequent.

Spinal tuberculoma is very rare form of nonosseous spinal tuberculosis, may occur in extradural (64\%), intramedullary $(8 \%)$, or intradural-extramedullary $(1 \%)$ spinal compartment. ${ }^{[2]}$ Intradural-extramedullary tuberculous mass is uncommon and can present as a single, dural-based ring enhancing lesion or rarely as a long segment enhancing soft tissue intradural mass lesion and such en plaque variety may mimic meningioma radiologically. ${ }^{[3]}$ Clinically, intradural-extramedullary tuberculoma commonly presents with feature of compression of nerve roots and spinal cord; however, it may rarely be associated with arachnoiditis ${ }^{[2,3]}$ and usually detected as a result of paradoxical response to antitubercular medication. ${ }^{[4]}$ It can rarely be associated with concurrent syringomyelia. ${ }^{[4]}$ However, association of intradural tubercular mass in association with peripheral osteoarticular tuberculosis of nonweight wearing elbow joint is extremely unusual. In detailed PubMed search, author could not get any report describing elbow joint tuberculosis associated with intradural-extramedullary mass of tuberculous origin; hence, current report represents first of its kind in the Western literature. ${ }^{[5]}$

The appearance of tuberculoma on MRI depend on the stage of development, maturity of rim, and content and the tuberculoma can be classified into three groups: first noncaseating lesion, others are caseating variety with central solid component and final group comprising

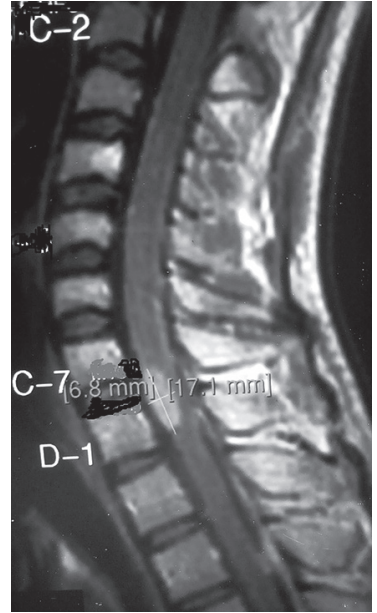

Figure 3: Gadolinium-enhanced magnetic resonance cervicodorsal spine, coronal section image showing conglomerate ring lesion at C7-D1 cervicothoracic junction suggestive of tuberculoma

caseation with totally liquid content. ${ }^{[6]}$ The noncaseating tuberculoma commonly demonstrates hypointense signal on T1-weighted images and hyperintense on T2-weighted images and homogenous nodular enhancement on contrast, while caseating granuloma with solid center shows hypointense to isointense on $\mathrm{T} 1$ image and typical hypointense on $\mathrm{T} 2$ images and only shows contrast enhancement of peripheral rim. The final group caseation with totally liquid content demonstrate hypointense signal on T1-weighted image and hyperintense on T2-weighted with peripheral hypointense rim, and on contrast study rim shows enhancement. ${ }^{[7]}$ A variable degree of vasogenic edema usually accompanies the lesion and is relatively more prominent in the early stages.

The elbow joint involvement observed in $1 \%-5 \%$ of the patients with skeletal system involvement is rare but reported ${ }^{[8-11]}$ A plain X-ray of elbow joint is reliable in diagnosis and during follow-up period to monitor response. Radiologically, the tubercular arthropathy in early stage of synovitis and arthritis shores periarticular demineralization, widening of joint space due to joint effusion, and early subchondral erosion..$^{[9-11]}$

As the tubercular arthropathy advanced, characteristic feature includes stage of erosion and in progressively enters into destruction phase producing narrowing of joint spaces by destruction of the articular cartilage, subchondral erosion and destruction, pathological subluxation and dislocation and ultimately produces fibrous ankylosis. Our case typically demonstrated advanced phase of elbow joint tuberculosis with gross dislocation and presence of the fibrous ankylosis. However, bony ankylosis is not seen in osseoarticlular tuberculosis but typically present in pyogenic arthritis. ${ }^{[7-11]} \mathrm{A}$ triad of characteristic feature observed on 
plain X-ray include periarticular osteoporosis, peripheral osseous erosion, and gradual reduction of the joint space on serial imaging is also known as Phemister's triad. ${ }^{[8,9]}$

Diagnosis of osteoarticular tuberculosis is difficult to challenging clinically. ${ }^{[7,8]}$ The most important is high degree of clinical suspicion and detailed history and clinical examination. Usually symptoms are nonspecific and early stage may be associated with, swelling of joint redness and painful movement of the joint and limitation of motion can be seen in advanced stage. ${ }^{[7,9]}$ However, typical features such as evening rise of temperature, malaise, anorexia, night sweats, and weight loss may not always present. The raised erythrocyte sedimentation rate may be helpful, however, not diagnostic. ${ }^{[12]}$ Usually the presence of acid-resistant staining of the joint fluid aspirate and positivity cultures of $M$. tuberculosis bacilli are diagnostic however in the difficult cases, a tissue biopsy is considered important for diagnosis confirmation. ${ }^{[6,9]}$

Our case had typical intradural tubercular lesion associated with advanced tuberculosis. Treatment of tuberculous arthropathy if started in early phase may respond to antituberculous medication; however, advanced stage needs surgical intervention including arthrodesis as the current case is awaiting arthrodesis of elbow joint under orthopedician care. The management of intradural lesion of tuberculosis origin may respond to appropriate antituberculous medication. However, unresponsiveness to medication or fresh appearance of neurological deficit or paradoxical response to antituberculous therapy may warrant surgical excision and laminectomy with gross total excision was carried out in our cease. ${ }^{[8,9,12]}$ However, junctional region mass may elude early diagnosis as current cases also.

Involvement of tuberculosis in upper limb joints is uncommon, usually affects larger weight-bearing joints such as hip, knee, and ankles joints. As our cases had recrudesces of pulmonary tuberculosis, intradural-extramedullary mass with elbow joint dislocation and fibrous ankylosis represents uncommon pathological association in the western literature, representing such association as first of its kind. Again old medical wisdom is correct, first try to link multiple pathology as a consequence of common initiating single pathology. Again author recommends that a patient with multiple pathology should undergo appropriate neuroimaging study and usually single pathology may turn out be to be culprit and before leveling multiple pathological diseases, extreme degree of caution is advocated.

\section{Financial support and sponsorship}

Nil.

\section{Conflicts of interest}

There are no conflicts of interest.
Guru Dutta Satyarthee

Department of Neurosurgery, Neurosciences Centre, AIIMS,

New Delhi, India

Address for correspondence: Dr. Guru Dutta Satyarthee, Department of Neurosurgery, Neurosciences Centre, AIIMS,

Room No. 714, New Delhi, India.

E-mail: duttaguru2002@yahoo.com

\section{REFERENCES}

1. Grosskopf I, Ben David A, Charach G, Hochman I, Pitlik S. Bone and joint tuberculosis - A 10-year review. Isr J Med Sci 1994;30:278-83.

2. Roca B. Intradural extramedullary tuberculoma of the spinal cord: A review of reported cases. J Infect 2005;50:425-31.

3. Shim DM, Oh SK, Kim TK, Chae SU. Intradural extramedullary tuberculoma mimicking en plaque meningioma. Clin Orthop Surg 2010;2:260-3.

4. Gul S, Celebi G, Kalayci M, Acikgoz B. Syringomyelia and intradural extramedullary tuberculoma of the spinal cord as a late complication of tuberculous meningitis. Turk Neurosurg 2010;20:561-5.

5. Yazici A, Kayan G, Yaylaci S, Demir MV, Karakeçe E, Tamer A, et al. Tuberculous arthritis of the elbow joint: A case report. Eur J Rheumatol 2016;3:142-3.

6. Jinkins JR, Gupta R, Chang KH, Rodriguez-Carbajal J. MR imaging of central nervous system tuberculosis. Radiol Clin North Am 1995;33:771-86.

7. Gupta RK, Jena A, Sharma A, Guha DK, Khushu S, Gupta AK. MR imaging of intracranial tuberculomas. J Comput Assist Tomogr 1988;12:280-5.

8. Golden MP, Vikram HR. Extrapulmonary tuberculosis: An overview. Am Fam Physician 2005;72:1761-8.

9. Ludwig B, Lazarus AA. Musculoskeletal tuberculosis. Dis Mon 2007;53:39-45.

10. Novatnack ES, Protzman NM, Kannangara S, Busch MF. Elbow Mycobacterium tuberculosis in America. J Glob Infect Dis 2015;7:44-5.

11. Domingo A, Nomdedeu M, Tomás X, García S. Elbow tuberculosis: An unusual location and diagnostic problem. Arch Orthop Trauma Surg 2005;125:56-8.

12. Dawar P, Satyarthee GD, Sharma BS. Total Resolution of large scalp swelling due to calvarial tuberculosis with medical management only: Case report and review of the literature. Turk Neurosurg 2015;25:313-6.

This is an open access article distributed under the terms of the Creative Common Attribution-NonCommercial-ShareAlike 3.0 License, which allows others to remix, tweak, and build upon the work non-commercially, as long as the author is credited and the new creations are licensed under the identical terms.

\begin{tabular}{|l|l|}
\hline \multicolumn{2}{|c|}{ Access this article online } \\
\hline Quick Response Code: & Website: \\
\hline & www.ruralneuropractice.com \\
\cline { 2 - 2 } & \\
\hline
\end{tabular}

How to cite this article: Satyarthee GD. Advanced elbow joint tuberculosis with Cervicothoracic junction extramedullary tuberculoma in a school boy: Unusual association. J Neurosci Rural Pract 2017;8:S147-9.

(C) 2017 Journal of Neurosciences in Rural Practice | Published by Wolters Kluwer - Medknow 